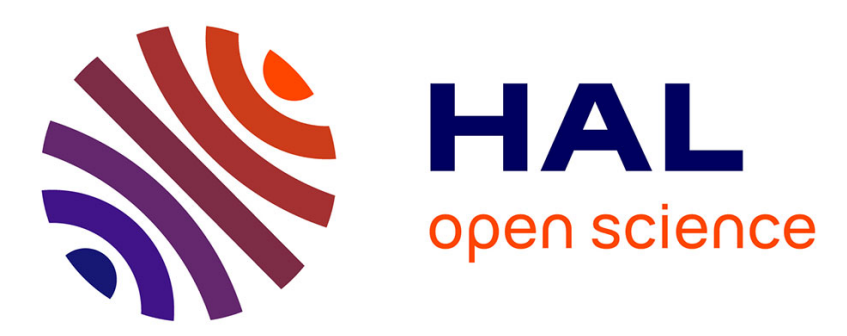

\title{
Automated Signal Analysis for Chirp Reinjections and SAR Images
}

Joseph Martinot-Lagarde, Hélène Oriot, Hubert Cantalloube

\section{To cite this version:}

Joseph Martinot-Lagarde, Hélène Oriot, Hubert Cantalloube. Automated Signal Analysis for Chirp Reinjections and SAR Images. RADAR 2019 (International Radar Conference), Sep 2019, Toulon, France. hal-03306706

\section{HAL Id: hal-03306706 https://hal.science/hal-03306706}

Submitted on 29 Jul 2021

HAL is a multi-disciplinary open access archive for the deposit and dissemination of scientific research documents, whether they are published or not. The documents may come from teaching and research institutions in France or abroad, or from public or private research centers.
L'archive ouverte pluridisciplinaire HAL, est destinée au dépôt et à la diffusion de documents scientifiques de niveau recherche, publiés ou non, émanant des établissements d'enseignement et de recherche français ou étrangers, des laboratoires publics ou privés. 


\section{Automated Signal Analysis for Chirp Reinjections and SAR Images}

\author{
Joseph Martinot-Lagarde \\ $D E M R$ \\ ONERA \\ Salon-de-Provence, France \\ joseph.martinot-lagarde@onera.fr
}

\author{
Hélène Oriot \\ $D E M R$ \\ ONERA \\ Palaiseau, France \\ helene.oriot@onera.fr
}

\author{
Hubert Cantalloube \\ DEMR \\ ONERA \\ Palaiseau, France \\ hubert.cantalloube@onera.fr
}

\begin{abstract}
The airborne radar team at ONERA developed tools to help check the data quality of its ever evolving radars, two of which are being presented here. The first one analyzes chirp reinjections to validate the proper functioning of the radar system, showing graphs of temporal and spectrum data over slow and fast time as well as quantitative measurements of the signal characteristics. The second one extracts information from SAR images and corner reflectors to quantify the quality of the data, geolocalisation and calibration precision, including experimental automated algorithms for parameter estimation. The promising results are to be improved and extended to cover more aspects of scientific data validation.

Index Terms - signal, quality, radar, chirp, SAR
\end{abstract}

\section{INTRODUCTION}

Science should be reproducible. The signal quality of a system must be verified before any study can be based on it. This is especially true for radar systems where the raw data is not usable until some processing is performed, so the quality of the data is not visible at first sight. At ONERA airborne radar research team, new radar configuration are regularly tested, the system is constantly evolving to address the needs of multiple research topics. The verification of the proper operation of such a system is not an easy task and usually done by hand to be able to adapt to every setup. To address this problem, ONERA developed generic tools to be able to check the recorded data quickly and extensively. Two main programs will be presented herein: chirp and SAR (Synthetic Aperture Radar) image analysis.

\section{PRINCIPLES}

Usually, signal checks are made manually, by plotting profiles or spectrums of the signal data, and checking that nothing seems out of the ordinary. This process is very repetitive and often boring, highly dependent on the operator and usually non exhaustive. There can be many advantages to automated tools such as determinism, speed, traceability, but they have to be programmed accordingly. We define a few general principles to be followed while developing the tools:

- The execution should be fast, typically a few seconds, up to one minute at worst. This way the analysis can be run systematically on every recording.

- There are as few input parameters as possible. This simplifies both the code and the usability, and keeps the output format similar between different analysis, enabling easy comparison to check for evolution in the data.

- Script parameters, data configuration and environment are stored along the results, for traceability and ease of rerunning the exact same analysis.

- Every graph is normalized and is as quantitative as possible. All useful variables should be measured or computed and displayed to the operator.

- Analysis are ordered in a logical order so that a problem detected in a later part does not invalidate the previous results.

Last but not least, these tools are not meant to replace the operator but to help him scrutinize the data. As configurations and requirements vary on a case-by-case basis, the tools will not try to determine themselves if the data is correct or not. It is up to the operator to decide and the tools job is to provide him with enough information.

\section{A. Formats and Programming}

The analysis output is presented as a single static HTML page containing plots, images, tables and text (as shown as examples later). This format presents many advantages not offered by more commonly used documents such as DOC or PDF: there is no need to manage page breaks since the page length is practically infinite, the document can easily be seen online as well as offline in any web browser, and the web is so dynamic nowadays that many tools are available to easily build such documents. One notable downside is that an HTML page with embedded images is not a single file on disk, thus it is not immediately transferable by e-mail.

The programs are mostly written in python, with a few modules in $\mathrm{C}$ for performance or reuse of existing code. Scientific python packages are used in addition to the standard library: Numpy and Scipy are heavily used for the computations, plots are generated by Matplotlib, images are edited with Pillow and Cython is used as an interface to $\mathrm{C}$ and to speed up computations. Reports are written in restructuredText format, which is then compiled by docutils to form the final HTML page. 


\section{CHIRPS}

The most commonly used signal for SAR imaging is the linear frequency ramp called chirp. Its basic formula is very simple:

$$
x(t)=\sin \left[2 \pi\left(f_{0} t+\frac{f_{1}-f_{0}}{2 T} t^{2}\right)\right], t \in[0, T]
$$

where $f_{0}$ is the starting frequency, $f_{1}$ is the final frequency and $T$ is the duration of the chirp.

In a real radar system this chirp is generated and then passes through multiple elements (cables, transitions, filters, isolators, amplifiers, attenuators, combiners, dividers, up/down mixers...). These elements distort the signal and analysis of these imperfections give information about the state of the system. Some distortions can be easily compensated in postprocessing, while others have to be fixed in the radar system itself.

To check the state of the system before or during the actual radar measurements, reinjection data are recorded. The chirp signal is generated but instead of being sent to the antenna, it switches to an internal circuit that returns it directly to the reception chain. This way the output signal contains information about the transfer function of all elements it passes by.

The analysis program takes two arguments: the file name of the raw data containing repeated chirp impulsions (real or I/Q complex), and a text file describing the wave form (analog frequencies, frequency rates in fast and slow time, signal length...). It is adapted only to data from pulsed radars, continuous wave radars are out of scope here.

\section{A. Fast Time (Range)}

The very first thing to look at is the fast time temporal amplitude, compared to the theoretical parameters. It is not possible to check the whole record here, thus only the first impulsion is plotted (Fig.??) (stability over slow time is checked later). Information is given to check that the signal corresponds to the parameters, duration and position mostly. There is usually a time shift due to electronic delay inside the system, with typical values less than $1 \mathrm{~s}$. It is measured here roughly with a one sample precision by correlation of the amplitude with a rectangular signal of the chirp's duration. This method was preferred over edge detection to avoid being parasited by noise peaks. Hilbert transform to compute the signal envelope has been considered but not tested yet.

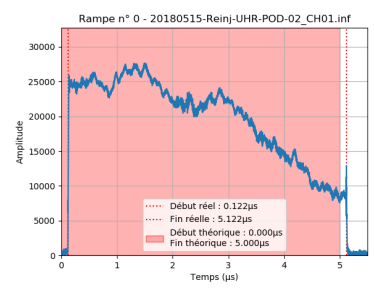

Fig. 1. Fast-time amplitude profile of a reinjected chirp
The other information given here is the measurement of signal level, including saturation detection. The vertical axis limits of the graph correspond to the range of values that the digital signal can take, so that the relative signal level can be easily viewed. In our systems, data are usually recorded in 10 , 12 or 16 bits corresponding to ranges of 1024, 4096 or 65536 . The histogram is also plotted to have a complementary display of the value distribution.

\section{B. Band Variations}

Distortion of the signal can already be visible in the temporal data, but it is best viewed in the frequency domain. The full spectrum is shown, normalized by $1 / N$ ( $N$ being the number of data samples) to maintain the amplitude level. The graph also shows the theoretical frequency range to check that the signal band is not shifted or truncated.

To have a mode precise measurement, the spectrum is actually averaged over multiple pulses (128 by default) in two different ways. First is the incoherent mean, which averages the amplitude of the different spectrums. It smooths the noise without changing its level. Next is the coherent mean, where the complex spectrums are averaged. In this case random noise will have a random phase and its average will be lower, while coherent signal and coherent noise will keep their level. The comparison of these two kind of averages shows what kind of noise is present in the signal. The detection of coherent noise is especially important because it usually is the source of artifacts in the final product. To have quantitative results, the RMS (Root Mean Square) level of the signal and each of the noise averages is also displayed.

The full spectrum shows the noise outside of the signal band, but inside the useful band the signal hopefully dominates. This is where we use a characteristic of the chirp: there is a linear relationship between time and frequency in its formula. If we divide the chirp temporally, the spectrum will be cut accordingly, as can be shown using equation (??). The chirp has been divided here in three temporal parts, and the spectrum for each part is plotted independently with the same averaging modes as the full spectrum presented before. Any coherent noise inside the useful band indicates an imperfection in the signal, typically harmonics or shifted replicas. This method is relatively limited, as it still doesn't show artifacts in the same band as the signal, and it usually gives very few information about the problems brought out. It is however useful as indices to be aware of in the subsequent analysis.

\section{Range Compression}

Now that all the information has been extracted from the raw signal, the range compression is computed in frequency domain, which enables more advanced analysis.

The spectrum was analyzed globally in the previous part, but now we can focus on the useful signal band. To be able to analyze the amplitude precisely, a range compression technique is used where instead of the standard complexconjuguate replica, the inverse of the chirp spectum is used. This way the phase is the same but the Gibbs effect on 

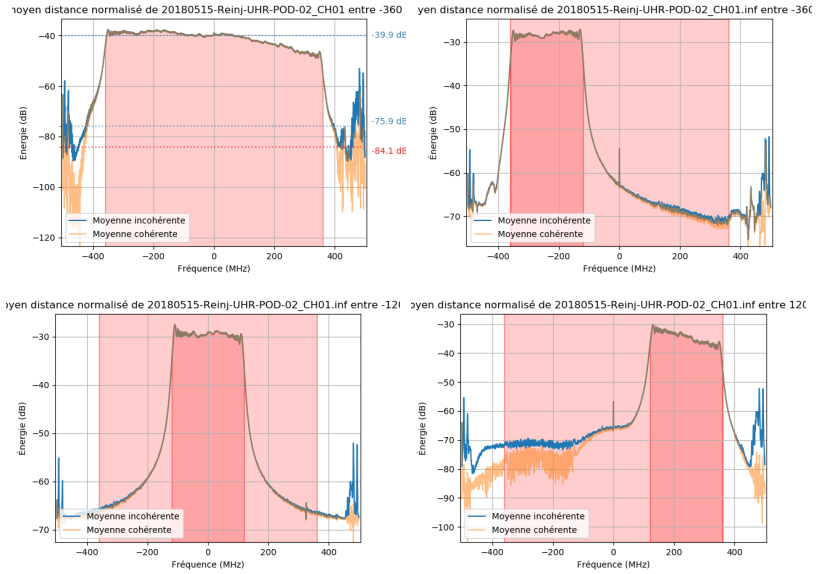

Fig. 2. Full and split short-time spectrums. On the left images the fat lines inside the useful bands are indications of noisy data (but very low level).

the amplitude is negated, which results in a theoretical flat spectrum in amplitude and phase. This method highlights the deterioration introduced by the hardware on the edges of the useful band.
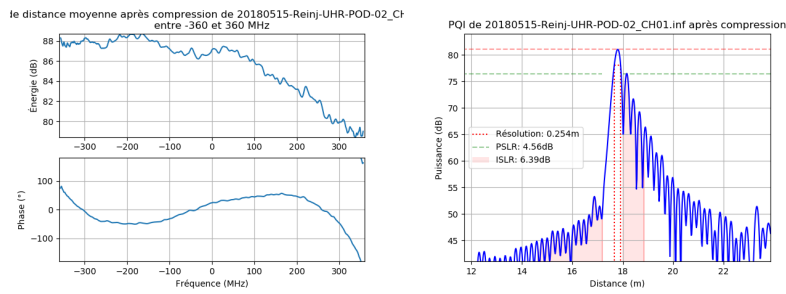

Fig. 3. Spectrum and temporal data (zoom) after range compression. The distortion is typical of band-pass filters and will be compensated during the processing.

The spectrum phase after range compression is composed of a linear ramp directly linked to the delay, and additional distortions by the radar system. Compensation of the mean slope gives a precise measurement of the electronic delay inside the system with a precision down to the phase noise, which is significantly better that the sample precision of the temporal method used earlier. When reinjections are recorded during actual measurement, this value is used to compensate precisely for synchronization variations or temperature shifts in the system, which removes the need of calibration devices on imaged sites for SAR images. Once the slope is removed, the residual is plotted to show the phase response of the system. This measurement is used to numerically compensate the defaults of the systems with a high precision.

Of course range compression is also useful to analyze the temporal signal, as the artifacts detected will be directly visible on final products. The temporal (or range) signal is ideally a sinus cardinal. With a rectangular window the resolution and PSLR (Peak Side Lobe Ratio) are compared to the theory, as well as the far lobes level. To better detect signal leaks, harmonics or shifted replicas, a hamming window is later applied to lower the side lobes and highlight lower artifacts. The two kinds of averaging presented in the previous part are applied, this enables to differentiate between the noise level and the far lobes.

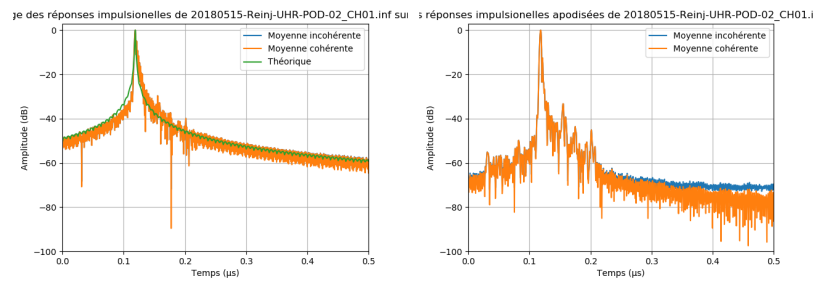

Fig. 4. Full temporal data after range compression, with rectangular and hamming windowing. The latter shows more artifacts.

\section{Slow Time (Azimuth)}

Radar needs to be coherent between pulses to be able to use doppler techniques or azimuth compression. The phase noise over slow time is a critical aspect that is last checked once everything is correct in fast-time. This is the slowest part of the analysis since it has to use information from the whole signal data. The general principle is that the peak of the range signal after compression contains enough information about the stability of a pulse. The peak position of the first pulse is measured as a reference, and the data considered here consists of the sample at this position for each recorded pulse. This is a 1D array of complex values, one for each slow time record.

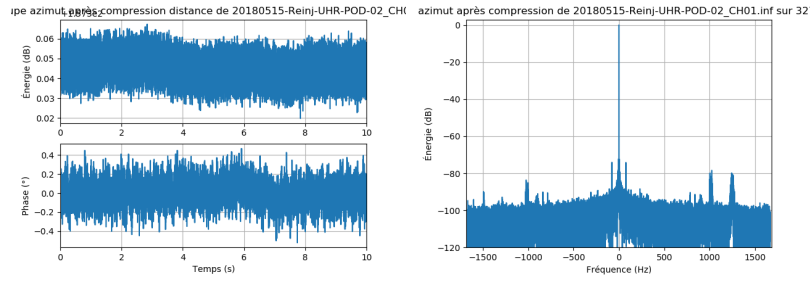

Fig. 5. Temporal and spectral slow-time data

The first information plotted here is the temporal evolution of the amplitude and phase. Any peak or parasite or strong variation indicates a problem: stability, synchronization, sample shift, firmware bug... Another artifact that can appear here is a drift of the values, that can be due to temperature variation or absence of synchronization with the typical $10 \mathrm{MHz}$ reference clock.

The most detailed information comes from the spectrum of this data. The low frequency noise is an indication of the temporal jitter. Spikes are usually the most embarrassing problems, they are the equivalent of leaks in the range profile and often translate into phantom targets in the final products. One of the common origins of the spikes are power sources or bad quality frequency oscillators. The ideal data consists in a constant value, thus the spectrum is normalized so that the null frequency is at $0 \mathrm{~dB}$. This way the comparison between different configurations is easily done. 


\section{SAR IMAGE}

The previous section was all about analyzing raw signal, but the same principles can be applied to mode refined data. High quality airborne SAR (Synthetic Aperture Radar) image production is one of the many capabilities of the radar department, and a perfect candidate for automated quality assessment. Multiple aspects can be evaluated, but precise measurements need calibration reflectors on the imaged zone, typically a set of corner reflectors. The general principles listed at the top of this article still hold, with a little twist: the report will first show a summary for the different analysis, then a detailed explanation for each reflector is added as an annex.

The analysis program takes only two inputs: the image itself in slant geometry (containing many auxiliary data in the header) and a text file containing the name and the GPS position of each calibration reflector to analyze.

\section{A. General Information}

The first part is not so much an analysis but rather a collection of all useful characteristics of the SAR image considered, extracted or computed from the header. First is a downscaled multilooked JPEG image of the considered SAR image, both for display and as a base for later analysis. The multilook factor is simply set so that the resulting image will fit in a standard HTML page, arbitrarily set here to 1000 pixels. The slowest part of the whole analysis is actually the production of the JPEG representation, which can obviously not be skipped.

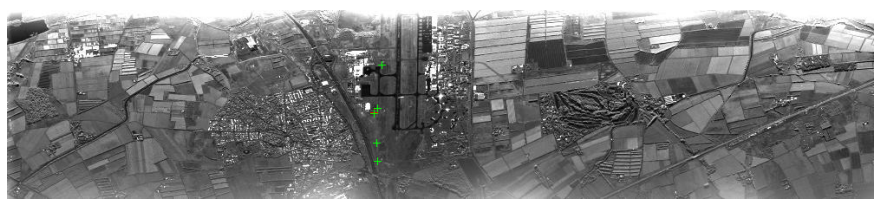

Fig. 6. SAR image over a calibration area near Garons (south of France). Green crosses indicate the position of corner reflectors (4 trihedrals and one dihedral)

A link to an auto-generated KML file is inserted. This file can be displayed in common geographic programs such as Google Earth, it shows the trajectory and the position of the image on the map. It doesn't include the image itself since it is in slant geometry, not projected into a map geometry.

Tables with useful numerical information are added such as range, swath, depression and incidence angles, azimuth length, integration angle, theoretical resolution... The input parameters are also displayed here fore reference.

\section{B. Geolocalization}

The first actual analysis is about the geolocalisation precision according to the target positions given as input parameter.

Before actually measuring the potential positioning error, an algorithm matches the theoretical positions of the targets with surrounding local maxima. Special care has been taken so that close theoretical positions will not result in the same detected point. A rectangular search area is defined around each theoretical position, which is by default $50 \mathrm{~m}$ in range and $5 \mathrm{~m}$ in azimuth (range position can be initially less precise due to varying electronic delays). For each search area, we compute a mask keeping only positions nearest to the center that any other area center, to ensure that the search area for each reflector never overlap. The pixel with a maximum amplitude over the mask is selected as the measured position after oversampling by a factor 16 to get precise values.

A zoom over the zone containing the calibration devices (such as corner reflectors, Luneburg lenses, top hat or transponders) is displayed, with colored crosses indicating the position of the theoretical coordinates and the corresponding positions picked by the algorithm presented before. The operator has to check that the detection matches correctly, since most subsequent analysis will be applied to these positions.

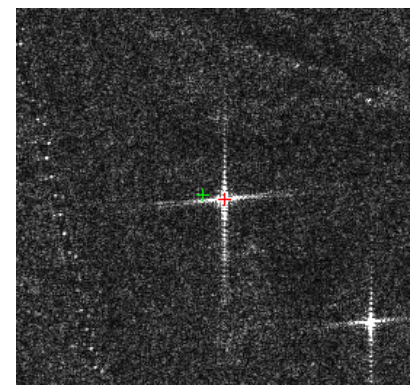

Fig. 7. Gelocalization of a corner reflector, with input coordinate in green and detection in red. The offset is $-0.34 \mathrm{~m}$ in range (vertical) and $-2.3 \mathrm{~m}$ in azimuth (horizontal).

Now that the input and measured positions are known, a table is displayed with a comparison of the values in different units: pixels, meters, and equivalent time difference (fast-time for range and slow-time in azimuth). The mean of these errors are also displayed which serves as a first correction value that can be used to compensate for the error. These values have to be taken with care, because they hold both the position error from the computation parameters and the GPS measurement system. A surveyor specialist can yield position errors down to $4 \mathrm{~cm}$, but a more standard device has a typical precision around $1 \mathrm{~m}$.

\section{Image Quality}

The theoretical response of a corner reflector is a cardinal sine cardinal in both range and azimuth directions. We run the same kind of analysis as with the chirp reinjection after range compression from the previous part to estimate the resolution and PSLR and compare them to the theoretical values. Since the reflectors are placed above clutter it is not possible to check for far lobes.

In the range direction the band distortion is estimated and averaged over each calibration target. The reinjection analysis was previously computed over a lot more points (many thousands compared to less than 256 on the image), but it did not include some parts of the system such as the antennae and some high-power circuits. This measurement on 
the image has less details but contains the residual distortions to be compensated, so it has to be taken into account. This automatic feature is still experimental but once consolidated it could be directly used for post-processing band compensation.
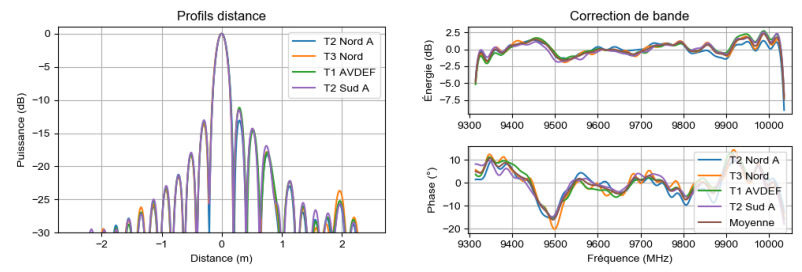

Fig. 8. Range profile and spectrum over 4 corner reflectors

In the azimuth direction the distortion comes from the inexact movement compensation of the phase center of the antennae. It can be due to the trajectory itself, in this case the error varies along the image. It can also come from wrong estimation of computation parameters such as electronic delays, which may change from acquisition to acquisition, though they only marginally drift during a given acquisition. The current analysis only includes a rough autofocus algorithm to estimate the refocalisation potential. As for the band estimation in the range direction, this algorithm is still experimental but could be replaced by the full blown algorithm used manually so that the analysis would compute directly the corrected parameter.

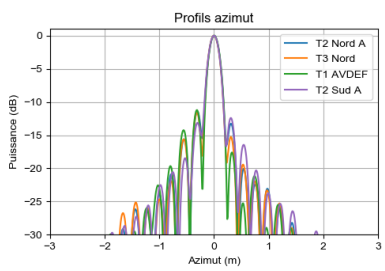

Fig. 9. Azimuth profile for 4 corner reflectors

\section{Calibration}

Calibration is an important feature of a SAR image. This analysis has two modes: it can either check the correctness of a calibrated image, or attempt to calibrate a non calibrated image. The underlying algorithm is actually the same (give or take a subtraction in log-scale), only the output display will be different. The calibration obviously relies on the calibration reflectors disposed on site and detected in the geolocalisation section above. The calibration reflectors used have standardized names corresponding to the size of the trihedral edges. An analytic formula gives the maximum RCS (Radar Cross Section) as a function of the wavelength $\lambda$ and length $d$ of the edges:

$$
R C S=\frac{4 \pi d^{4}}{3 \lambda^{2}}
$$

The ratio between the amplitude value on the image and the theoretical value of the RCS gives the calibration factor to apply on the image. This configuration factor can be applied to other images if taking into account the potential changes of the attenuation values of the hardware system made to adapt the signal level to the different image scenes.

The calibration computed here is rather crude. For example it doesn't take into account the exact direction of the wave arriving on the corner reflector. As with other analysis, more work is needed to be able to use the program for automated calibration in production.

To be able to measure NRCS (Normalised Radar Cross Section), the calibration surface has to be estimated on the corner reflectors. Two different methods have been implemented. The simplest one merely consists in computing the product of the range and azimuth resolutions, with an additional factor depending on the windowing ( 0.886 for the rectangular window). The other way is to get back to the definition of the calibration surface: it is the ratio between the energy over the surface and the RCS. The problematic part is to estimate the energy, since the signal of the reflector is mixed with the surrounding clutter. Here the energy is integrated over the first two side lobes in each direction, so that most of the energy is taken into account while the bias from the clutter signal is kept low.

The calibration factor and surface are computed for each target, then the mean is computed. If the image is calibrated, it is used to estimate the mean calibration error. If the image is not calibrated, the mean is used to calibrate the image.

Once the amplitude calibration and the calibration surface have been determined, it is possible to look for the $N_{e} \sigma_{0}$ (Noise equivalent reflectivity). An estimator for the $N_{e} \sigma_{0}$ is the lowest $\sigma_{0}$ value found on the image, which usually corresponds to shadows or still water areas. This is where the multilooked JPEG image is reused: instead of looking over the whole image (which would take a tremendous amount of time), the search is only done over the pixels with the lowest value. For each of these lower value pixels, a $256 \times 256$ pixels square is extracted from the original data, in which the minimum $\sigma_{0}$ over a $31 \times 31$ pixels square is selected. The final $N_{e} \sigma_{0}$ is the lowest value amongst the candidates. This measure is actually an upper bound of the real $N_{e} \sigma_{0}$ value, because the darkest areas are polluted by far side lobes from the targets and clutter around, but for practical purposes this measure is representative of the background noise of the image.

The last analysis is the estimation of the lobe aiming error in site. If multiple calibration reflectors are disposed on the image, they are usually spread in range. If we suppose that the calibration gap for each one of them comes from a lobe aiming inaccuracy, it is possible to use minimization techniques to find the lobe position that yields the lowest possible error. First a function is written to estimate the illumination offset for given geometry directions corresponding to each reflector and an aiming offset angle. This is used in another function that computes the global residual error given an aiming offset angle; this is the one that will be minimized. Few iteration will give an estimation of the best offset angle up to 1/100th of degree, which is more than enough for the calibration precision. This method is stable enough to have already been 
used to compensate for aiming inaccuracies in actual image production.

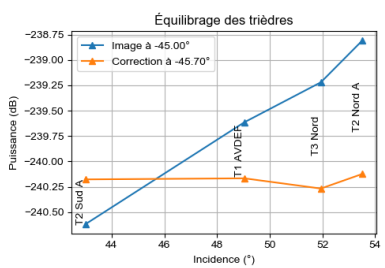

Fig. 10. Calibration factor over 4 corner reflectors before and after lobe aiming correction

\section{E. Details}

As stated in the introduction, the last part of the analysis contains detailed data for each of the calibration reflectors given in input: tables on numerical data about detection, geolocalisation, calibration, quality parameters and refocalisation presented before. There are also zooms and specific plots for each estimation.

Finally, useful extracts (errors, warnings and notes) from the log files generated by the SAR image processing are listed, for helping in debugging the numerous parameter values involved in the configuration.

\section{CONCLUSION}

This article has shown the usefulness of the different analysis for two different kind of data: chirp reinjections and SAR images. The programs are always work in progress: as techniques evolve, more and more precision is needed which translates into harder constraints on data quality. Automated analysis is invaluable to not get overwhelmed by the growing requirements. 